\title{
Afterload-related reference values for myocardial work indices
}

\author{
Qiancheng $\mathrm{Li}^{1 \dagger}$, Hui Wang ${ }^{2+}$, Haiyan Feng ${ }^{2}$, Tingfan Wu${ }^{3}$, Ying Yang ${ }^{2}$, Dongmei Gao ${ }^{2}$ and Lina Sun ${ }^{2 *}$
}

\begin{abstract}
Background: The novel noninvasive pressure-strain loop (PSL) is a reliable tool that reflects myocardial work (MW). Systolic blood pressure (SBP) is the only independent factor for MW indices. However, afterload-related reference values have not been previously reported. The aim of the present study was to establish reference values for MW parameters by wide range SBP grading.

Methods: We prospectively selected healthy individuals and subjects with $\mathrm{SBP} \geq 140 \mathrm{mmHg}$ at the time of study without myocardial remodeling. MW parameters were collected and the reference values achieved were grouped by SBP in 10-mmHg.

Results: Significant differences were noted among the SBP-groups for global work index (GWI) and global constructive work (GCW). The majority of statistical comparisons of the differences in GWI and GCW were significant at each SBP-group. With SBP ranging from 90 to $189 \mathrm{mmHg}$, the parameters GWI and GCW tended to increase linearly with afterload. Overall, the global wasted work (GWW) tended to rise as SBP was increased, but not all of the differences noted in GWW were significant for each SBP-group. Global work efficiency (GWE) remained stable across all SBPgroups, with the exception of a slight drop noted when it exceeded $160 \mathrm{mmHg}$.
\end{abstract}

Conclusions: The amount of MW but not the work efficiency varied greatly according to the different afterload. This finding cannot be ignored during clinical research or diagnosis and afterload-related reference values are required to make a reasonable judgment on the myocardial function.

Keywords: Myocardial work, Afterload, Reference values

\section{Background}

The novel noninvasive pressure-strain loop (PSL) is a reliable tool to reflect myocardial work (MW) in a variety of hemodynamic states by comparison with invasive experimental and clinical studies. It has shown a strong correlation with regional myocardial glucose metabolism by positron emission tomography (PET) [1-4].

The PSL algorithm has two advantages over strain measurements in evaluating left ventricular function.

\footnotetext{
*Correspondence: sunlina@jlu.edu.cn

${ }^{\dagger}$ Qiancheng Li and Hui Wang have equal contribution as first co-authors.

2 Department of Ultrasound, China-Japan Union Hospital of Jilin University, Changchun, China

Full list of author information is available at the end of the article
}

Firstly, the main limitation of strain imaging is load dependency [5]. An increase in afterload can lead to decreased strain giving rise to misinterpretation of the true contractile function, which in turn leads to false conclusions with regard to reduced myocardial function. However, MW takes into account deformation as well as afterload, potentially offering incremental value to myocardial function assessment. This advantage was proved by a canine experiment where a substantial decrease in longitudinal strain (LS) occurred, whereas myocardial work index (MWI) was unaltered during aortic constriction [6]. Secondly, MW parameters are calculated as integration over time of the strain rate obtained by differentiating the strain curve multiplied by the instantaneous left ventricular pressure (LVP). LVP was estimated by a original author(s) and the source, provide a link to the Creative Commons licence, and indicate if changes were made. The images or other third party material in this article are included in the article's Creative Commons licence, unless indicated otherwise in a credit line to the material. If material is not included in the article's Creative Commons licence and your intended use is not permitted by statutory regulation or exceeds the permitted use, you will need to obtain permission directly from the copyright holder. To view a copy of this licence, visit http://creativecommons.org/licenses/by/4.0/. The Creative Commons Public Domain Dedication waiver (http://creativeco mmons.org/publicdomain/zero/1.0/) applies to the data made available in this article, unless otherwise stated in a credit line to the data. 
surrogate of the LVP curve. Therefore, MW indices were used to address all the changes noted in the myocardial strain along with LVP changing during the cardiac cycle and provide more comprehensive information for evaluating left ventricular (LV) function. By contrast, LS, the most commonly used strain index, is used to assess the peak systolic strain value, but not the process of obtaining the peak strain. It is well known that LVP changes over time in the cardiac cycle. Therefore, the same strain occurring at different time periods during systole may achieve different MW measurements. The identical peak LS value, which occurs in early or mid-or end-systole may not correspond to identical myocardial work. However, this information cannot be represented by LS, nor can it be reflected in case of transient hypokinisis, akinesis or even paradoxical movement, which can affect the MW. In other words, different patients may present significantly different MW values even if they have the same LS and the same systolic blood pressure (SBP) (Fig. 1).

Therefore, theoretically, MW is the most comprehensive indicator of left ventricular myocardial function. Previous studies have reported reference values for MW indices grouped by gender or age [7-9]. Despite these reference values, the wide variability of MW indices prevents the application of this technique in the routine clinical setting. Multivariable linear regression analysis for MW indices has shown that SBP rather than gender or age was the only independent factor following adjustment for confounders [7]. Therefore, it is not advisable to assess MW in the absence of SBP. In other words, a given MW level for a patient with SBP of $90 \mathrm{mmHg}$ has a significantly different meaning than the same $\mathrm{MW}$ for a patient with SBP of $180 \mathrm{mmHg}$. Given the superiority of $\mathrm{MW}$, an urgent need is required to establish reference values for MW parameters grouped by SBP. The present study recruited healthy subjects without hypertension and subjects in the early stage of hypertension without myocardial remodeling. To the best of our knowledge, the present study provided for the first time the reference values for MW indices based on SBP grading over a wide range to benefit the application of this new indicator in the routine clinical diagnosis.

\section{Methods \\ Subjects}

We prospectively selected subjects from healthy volunteers and participants who had received coronary tomography angiography (CTA) for health examination demonstrating no more than $50 \%$ coronary artery stenosis. The eligibility criteria for healthy subjects included the following: 1. normal 2-dimensional (2D) echocardiographic results without grade $>1$ regurgitation [10] 2. no history of diabetes mellitus, hyperlipidemia or cardiovascular disease 3. (1) SBP $<140 \mathrm{mmHg}$ at the time of study and no history of hypertension or (2) SBP $\geq 140 \mathrm{mmHg}$ at the time of study with history of hypertension $<1$ year

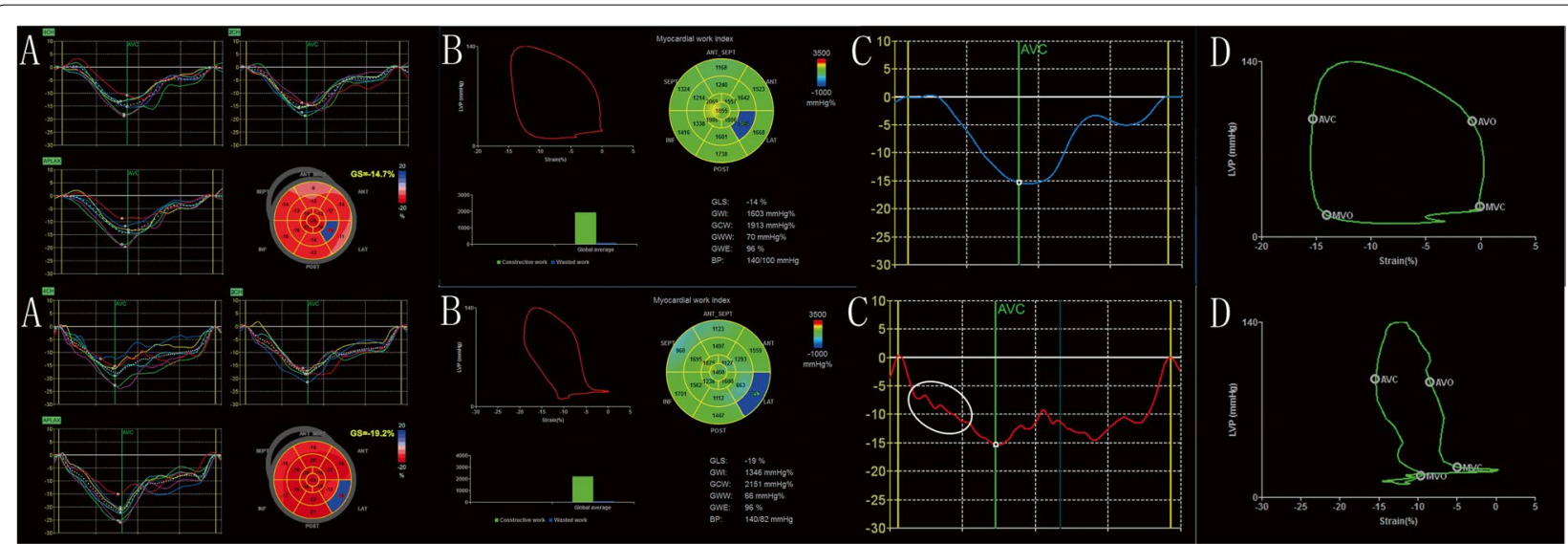

Fig. 1 Top line: data from a patient after adrenal tumor surgery with a history of hypertension (SBP > $180 \mathrm{mmHg}$ ) for more than 2 years. Bottom line: data from a patient with coronary heart disease. Coronary angiography demonstrated stenosis in the middle left circumflex branch (80\%) and in the proximal right coronary artery (70\%). (A, B in the top line) The patient's GLS value was $-14 \%$ with SBP of $140 \mathrm{mmHg}$ at the time of the study. The GWI was 1,603 mmHg\%. (A, B in the bottom line) This patient exhibited a higher GLS value of $-19 \%$ and a lower GWI (1,346 mmHg\%) at the same SBP level than the previously mentioned subject. Figure Cs and Ds explained these results. (C, $\mathbf{D}$ in the top line) The peak LS value of the middle segment of the lateral wall was $-15 \%$ and the MWI of the segment was 1,846 $\mathrm{mmHg} \%$. (C, $\mathbf{D}$ in the bottom line) Considerably lower MWI was noted in the basal segment of the lateral wall ( $853 \mathrm{mmHg} \%$ ) at the same peak LS and at the same SBP level. (C in the bottom line) It demonstrated that the strain curve with apparent transient hypokinisis, akinesis and even paradoxical movement in mid-systole (white oval) led to much lower MWI. However, this information could not be reflected by the LS value. SBP, systolic blood pressure; GLS, global longitudinal strain; GWI, global work index; LS, longitudinal strain; MWl, myocardial work index 
and without any myocardial remodeling based on normal 2D echocardiographic appearance and no history of antihypertensive medication administration. The present study was approved by the local ethics committees. All participants provided informed consent prior to the examination.

\section{Echocardiography}

Comprehensive transthoracic echocardiography (TTE) was performed according to the American Society of Echocardiography guidelines $[11,12]$ by an experienced sonographer using a Vivid E95 ultrasound system equipped with an M5S $3.5 \mathrm{MHz}$ and a $4 \mathrm{VC} 3.3 \mathrm{MHz}$ transducer (GE Vingmed Ultrasound, Horten, Norway). The patients were scanned in the left lateral decubitus position for optimal image quality.

\section{MW}

MW was measured from the PSL area using commercially available software Package (EchopacVersion 203, $\mathrm{GE}$ ), which was constructed from a surrogate of the LVP curve combined with LS acquired with speckle tracking echocardiography (STE), as proposed by Russell et al. [1]. 2D grayscale images from the apical four-chamber, two-chamber and long-axis views were acquired at $62-86$ frames/sec to enable LS analysis. In particular, the mitral and aortic valves were clearly displayed on the long-axis view. Three consecutive cardiac cycles for each view were acquired. For strain analysis, endocardial borders of all LV segments were clearly visualized throughout the whole cardiac cycle to assure optimal wall detection and tracking control. In addition, LV ejection fraction (EF) was achieved from apical four-chamber and two-chamber. The LVP was estimated in a noninvasive manner using peripheral brachial SBP, which was measured immediately following TTE with the patient being still in the left lateral decubitus position. When synchronized and normalized with valvular timing events, a surrogate of LVP versus time was generated. The LV strain and pressure data were subsequently synchronized by alignment of valvular timing events, which were all set manually on the long-axis view [13]. Instantaneous MW was quantified as the strain rate obtained by differentiating the strain curve multiplied by the instantaneous LVP. This instantaneous work was then integrated over time during systole (time interval from mitral valve closure through to mitral valve opening). The work performed during shortening in systole adding negative work during lengthening in isovolumetric relaxation represented constructive work $(\mathrm{CW})$ and work performed during lengthening in systole adding work performed during shortening in isovolumetric relaxation represented wasted work (WW). CW and WW were calculated for each LV segment, according to the 17-segment model. Global CW (GCW) and global WW (GWW) were calculated as the averages of the segmental values. Work efficiency (WE) was then expressed as $\mathrm{CW} /(\mathrm{CW}+\mathrm{WW}) \times 100 \%$ per segment and the global WE(GWE) as an average of all segmental values. The Global work index (GWI) was obtained as total work calculated from mitral valve closure to mitral valve opening, equal to the area of the PSL.

To assess the intra-observer and inter-observer reproducibility, 40 subjects were selected ( 5 random subjects from each SBP-group). For intra-observer variability, one set of images were collected in these individuals by an experienced physician (LN.S.). The time interval for the analysis of the same set of images in each subject should be at least one week and it was applied in a random order. For inter-observer variability, the other dataset was retained by another physician (HY.F.) using the same device in the same place. The images were analyzed by the same physician (HY.F.).

\section{Statistical analysis}

The normality of the distribution of continuous variables was assessed by the Kolmogorov-Smirnov test. Continuous data are reported as mean \pm standard deviation (SD) or median (interquartile range) as appropriate. The $95 \%$ confidence interval $(95 \% \mathrm{CI})$ was calculated as mean \pm 1.96 SDs for normally distributed continuous variables. The lowest (2.5th percentile) and highest (97.5th percentile) expected values for non-normally distributed continuous variables were estimated in 1,000 bootstrap samples to generate sampling distribution. The comparison across SBP groups was performed by the analysis of variance (ANOVA) for normally distributed variables with homogeneity of variance or by the Kruskal-Wallis test for variables without normal distribution or homogeneity of variance and specific group differences were tested by using a corrected alpha value $<0.002$. Scatter diagrams of GWI and GCW with SBP were plotted with trend curves obtained from locally weighted regression. Intra-observer and inter-observer variability was assessed using the Bland-Altman analyses and paired-samples $t$ test or Wilcoxon matched paired test were used to verify the significance of the bias. The SPSS statistical software (version 21.0; SPSS Inc, Chicago, IL, USA) and R studio (version 3.6.1; $\mathrm{R}$ studio, Boston, Massachusetts) were used for all analyses. $P<0.05$ was considered to indicate significant differences. 


\section{Results}

Of the 248 subjects included in the present study, 18 were excluded from further analysis due to poor image quality. Finally, a total of 80 men and 150 women were included. The demographic data of the population listed in Table 1 are grouped by SBP in 10-mmHg subgroups.

EF, GLS and MW indices grouped by SBP are presented in Table 2. No significant differences were detected for EF and GLS among SBP-groups. When SBP ranged from 90 to $189 \mathrm{mmHg}$, GWI and GCW tended to increase linearly with the afterload. Significant differences were noted among the SBP-groups for GWI and GCW. For GWI, the majority of the statistical comparisons of the differences at each SBP-group were significant with the exception of the comparisons for every two adjacent groups. For GCW, the majority of the differences between groups were statistically significant with the exception of the comparisons between the SBP 130-139 $\mathrm{mmHg}$ and 140-149 $\mathrm{mmHg}$ subgroups, the SBP $140-149 \mathrm{mmHg}$ and 150-159 $\mathrm{mmHg}$ subgroups and the SBP $150-159 \mathrm{mmHg}$ and $\mathrm{SBP} \geq 160 \mathrm{mmHg}$ subgroups (Table 2, Fig. 2).

Overall, GWW tended to rise with the increase of SBP, but not all of the differences in GWW were significant at each SBP group. The differences among the SBP $90-99 \mathrm{mmHg}, 100-109 \mathrm{mmHg}$ and $110-$ $119 \mathrm{mmHg}$ subgroups were not statistically significant. Non-significant differences were also noted among the SBP $100-109 \mathrm{mmHg}, 110-119 \mathrm{mmHg}$, 120-129 mmHg and $130-139 \mathrm{mmHg}$ subgroups. Similarly, non-significant differences were noted among the SBP 120-129 $\mathrm{mmHg}, 130-139 \mathrm{mmHg}, 140-$ $149 \mathrm{mmHg}$ and $150-159 \mathrm{mmHg}$ subgroups. The GWE

Table 1 characteristics of the population

\begin{tabular}{|c|c|c|c|c|c|c|c|c|}
\hline \multirow[t]{2}{*}{ characteristic } & \multicolumn{8}{|c|}{ SBP-groups $(\mathrm{mmHg})$} \\
\hline & $\begin{array}{l}90-99 \\
n=16\end{array}$ & $\begin{array}{l}100-109 \\
n=29\end{array}$ & $\begin{array}{l}110-119 \\
n=34\end{array}$ & $\begin{array}{l}120-129 \\
n=45\end{array}$ & $\begin{array}{l}130-139 \\
n=35\end{array}$ & $\begin{array}{l}140-149 \\
n=29\end{array}$ & $\begin{array}{l}150-159 \\
n=20\end{array}$ & $\begin{array}{l}\geq 160 \\
n=22\end{array}$ \\
\hline Men, n(\%) & $2(13 \%)$ & $5(17 \%)$ & 10(29\%) & $14(31 \%)$ & $17(49 \%)$ & $16(55 \%)$ & $7(35 \%)$ & $9(41 \%)$ \\
\hline Age(years) & $32 \pm 9$ & $39 \pm 14$ & $40 \pm 12$ & $39 \pm 12$ & $43 \pm 14$ & $49 \pm 13$ & $47 \pm 11$ & $44 \pm 13$ \\
\hline Height(cm) & $162 \pm 5$ & $162 \pm 5$ & $164 \pm 8$ & $165 \pm 8$ & $167 \pm 7$ & $168 \pm 9$ & $167 \pm 10$ & $168 \pm 8$ \\
\hline Weight(kg) & $57 \pm 6$ & $57 \pm 8$ & $63 \pm 11$ & $63 \pm 10$ & $70 \pm 12$ & $74 \pm 13$ & $67 \pm 12$ & $73 \pm 12$ \\
\hline Body surface area $\left(\mathrm{m}^{2}\right)$ & $1.60 \pm 0.10$ & $1.61 \pm 0.11$ & $1.68 \pm 0.18$ & $1.69 \pm 0.16$ & $1.79 \pm 0.17$ & $1.83 \pm 0.20$ & $1.75 \pm 0.20$ & $1.81 \pm 0.18$ \\
\hline Heart rate $\left(\min ^{-1}\right)$ & $76 \pm 7$ & $77 \pm 9$ & $79 \pm 8$ & $74 \pm 10$ & $76 \pm 9$ & $77 \pm 7$ & $78 \pm 8$ & $80 \pm 7$ \\
\hline $\operatorname{LVMi}\left(\mathrm{g} / \mathrm{m}^{2}\right)$ & $72 \pm 10$ & $73 \pm 14$ & $74 \pm 10$ & $74 \pm 11$ & $76 \pm 11$ & $79 \pm 10$ & $83 \pm 12$ & $86 \pm 11$ \\
\hline Fasting glucose (mmol/L) & $4.74 \pm 0.59$ & $5.02 \pm 0.56$ & $5.00 \pm 0.55$ & $4.80 \pm 0.53$ & $4.85 \pm 0.56$ & $4.81 \pm 0.43$ & $5.07 \pm 0.45$ & $5.04 \pm 0.49$ \\
\hline Total cholesterol (mmol/L) & $3.73 \pm 0.60$ & $3.89 \pm 0.56$ & $3.98 \pm 0.66$ & $4.09 \pm 0.61$ & $4.06 \pm 0.59$ & $4.27 \pm 0.60$ & $4.07 \pm 0.64$ & $4.22 \pm 0.51$ \\
\hline
\end{tabular}

Data are expressed as mean \pm SD. SBP systolic blood pressure; LVMi left ventricular mass index

Table 2 EF, GLS and MW indices grouped by SBP

\begin{tabular}{|c|c|c|c|c|c|c|c|c|}
\hline \multirow[t]{2}{*}{ parameters } & \multicolumn{8}{|c|}{ SBP-groups $(\mathrm{mmHg})$} \\
\hline & $90-99$ & $100-109$ & $110-119$ & $120-129$ & $130-139$ & $140-149$ & $150-159$ & $\geq 160$ \\
\hline $\mathrm{EF}$ & $63 \pm 4$ & $64 \pm 4$ & $64 \pm 5$ & $65 \pm 4$ & $65 \pm 5$ & $65 \pm 5$ & $64 \pm 6$ & $63 \pm 5$ \\
\hline GLS & $-20 \pm 2$ & $-20 \pm 1$ & $-20 \pm 2$ & $-20 \pm 1$ & $-21 \pm 2$ & $-20 \pm 1$ & $-20 \pm 2$ & $-19 \pm 2$ \\
\hline \multicolumn{9}{|l|}{ MW indices } \\
\hline GWI (mmHg\%) & $1599 \pm 182$ & $1743 \pm 150$ & $1883 \pm 228$ & $2049 \pm 182$ & $2223 \pm 260$ & $2364 \pm 233$ & $2481 \pm 211$ & $2690 \pm 342$ \\
\hline $95 \% \mathrm{Cl}$ & $1242-1956$ & 1449-2038 & $1436-2329$ & $1691-2406$ & $1713-2733$ & $1906-2821$ & 2067-2895 & $2021-3360$ \\
\hline GWC (mmHg\%) & $1792 \pm 163$ & $1985 \pm 153$ & $2143 \pm 200$ & $2329 \pm 164$ & $2523 \pm 247$ & $2706 \pm 191$ & $2868 \pm 241$ & $3080 \pm 321$ \\
\hline $95 \% \mathrm{Cl}$ & $1471-2112$ & $1684-2285$ & $1752-2535$ & $2007-2650$ & 2039-3007 & 2332-3080 & 2395-3341 & $2451-3708$ \\
\hline GWW (mmHg\%) & $42 \pm 14$ & $53 \pm 23$ & $54 \pm 26$ & $63 \pm 20$ & $76 \pm 33$ & $85 \pm 30$ & $88 \pm 31$ & $125 \pm 43$ \\
\hline $95 \% \mathrm{Cl}$ & $14-71$ & $8-98$ & $3-105$ & $25-102$ & $11-141$ & $26-143$ & $27-148$ & $40-209$ \\
\hline GWE (\%) & 97(96-98) & 97(96-98) & 97(96-98) & $97(96-97)$ & $97(96-97)$ & 96(95-97) & $96(95-97)$ & $95(94-96)$ \\
\hline $95 \% \mathrm{Cl}$ & $94-99$ & $94-99$ & $94-99$ & $95-99$ & $93-99$ & 93-99 & $94-98$ & $92-98$ \\
\hline
\end{tabular}

Data are expressed as mean \pm SD or as median (interquartile range)

$E F$ ejection fraction; GLS global longitudinal strain; $M W$ myocardial work; SBP systolic blood pressure; $C l$ confidence interval; GWI global work index; GCW global constructive work; GWW global wasted work; GWE global work efficiency 

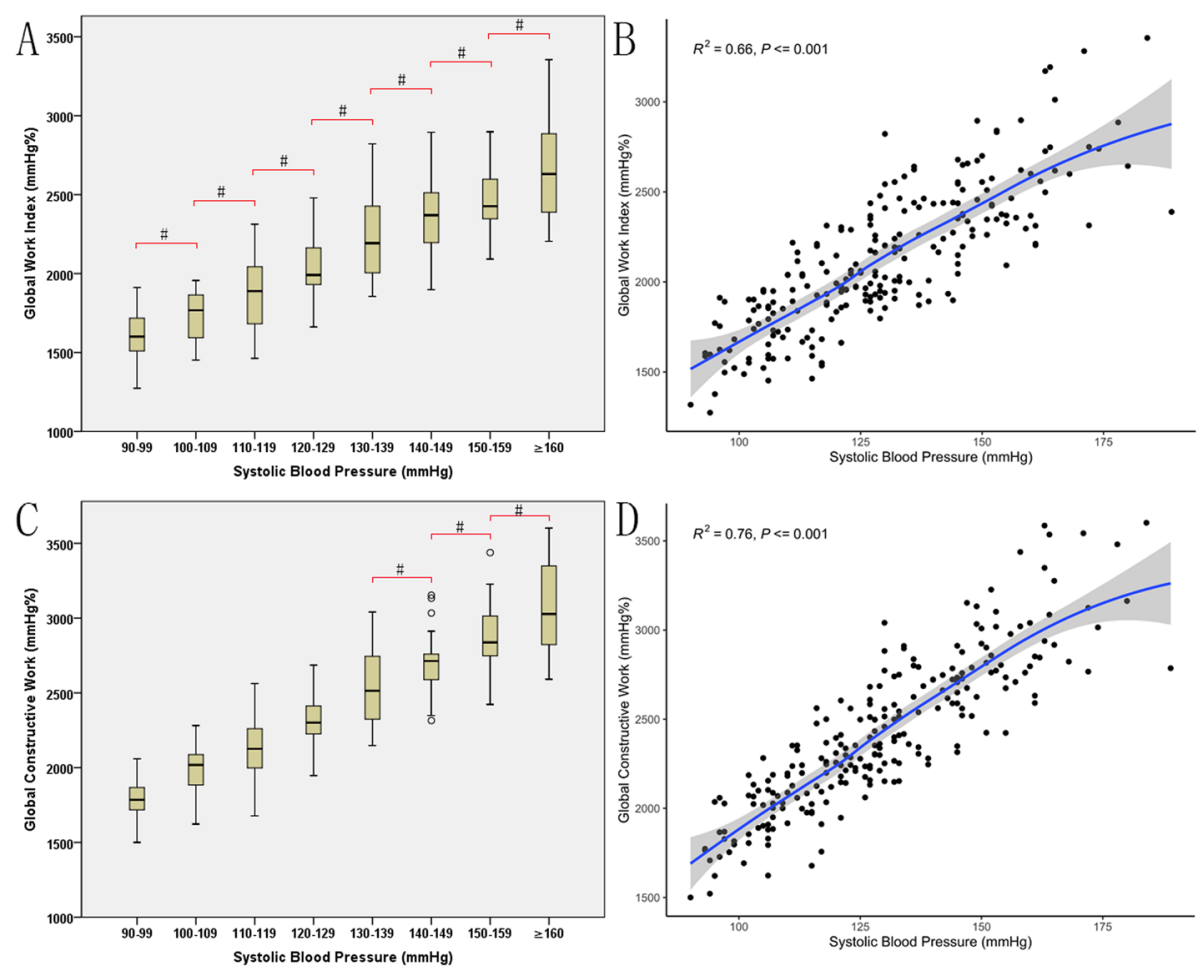

Fig. 2 Distribution of GWI and GCW values $(\mathbf{A}, \mathbf{C})$ and association of GWI and GCW with SBP (B, D). The curves were obtained from locally weighted regression. GWI, global work index; GCW, global constructive work; SBP, systolic blood pressure. ${ }^{P} P>0.002$, no significant difference

values remained stable among all SBP groups, with the exception of a slight drop noted when SBP exceeded $160 \mathrm{mmHg}$ (Table 2, Fig. 3).

Intra-observer and inter-observer analyses demonstrated optimal repeatability and reproducibility with regard to the MW parameters (Table 3).

\section{Discussion}

MW is derived from pressure-volume or pressurelength loops and it has been investigated for more than 40 years [14-17]. MW assessment was initially measured invasively during cardiac catheterization, which limited its widespread use in clinical practice. Russell et al. introduced recently a method of PSL (in $\mathrm{mmHg} \%$ ) for calculating MW non-invasively, which involved the combination of STE with LVP as estimated from brachial artery cuff pressure [1]. The accuracy of the novel method has been validated by subsequent studies [2-4]. A number of clinical researches have been performed within the last two years, since this technique was commercially available. To date, MW has been investigated with regard to cardiac resynchronization therapy (CRT) [18-23], in the
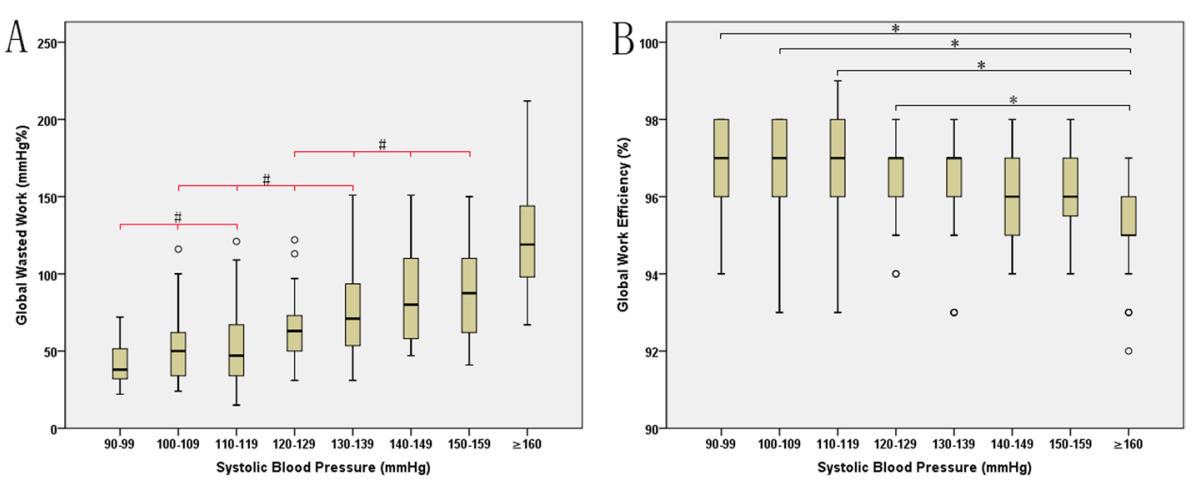

Fig. 3 Distribution of GWW (A) and GWE (B) values with SBP. GWW, global wasted work; GWE, global work efficiency; SBP, systolic blood pressure. ${ }^{\#} P>0.002$, no significant difference. ${ }^{*} P<0.002$, significant difference 
Table 3 repeatability and reproducibility of MW parameters

\begin{tabular}{lllll}
\hline variables & Value $_{1}$ & Value $_{\mathbf{2}}$ & Bias $_{2-\mathbf{1}}$ & $\boldsymbol{P}_{\text {-value }}$ \\
\hline Intra-observer & & & & \\
GWI (mmHg\%) & $2090 \pm 454$ & $2078 \pm 457$ & $-13 \pm 84$ & 0.350 \\
GCW (mmHg\%) & $2404 \pm 503$ & $2395 \pm 507$ & $-9 \pm 102$ & 0.583 \\
GWW (mmHg\%) & $76 \pm 34$ & $79 \pm 37$ & $3 \pm 24$ & 0.492 \\
GWE (\%) & $96(95-97)$ & $96(95-97)$ & $0(-1-1)$ & 0.826 \\
Inter-observer & & & & \\
GWI (mmHg\%) & $2090 \pm 454$ & $2071 \pm 440$ & $-19 \pm 90$ & 0.179 \\
GCW (mmHg\%) & $2404 \pm 503$ & $2375 \pm 493$ & $-29 \pm 97$ & 0.066 \\
GWW (mmHg\%) & $76 \pm 34$ & $77 \pm 38$ & $1 \pm 27$ & 0.733 \\
GWE (\%) & $96(95-97)$ & $96(95-97)$ & $0(-1-1)$ & 0.472 \\
\hline
\end{tabular}

Data are expressed as mean \pm SD or as median (interquartile range)

$M W$ myocardial work; GWI global work index; GCW global constructive work; GWW global wasted work; GWE global work efficiency

diagnosis of different categories of coronary heart disease [6, 24-28] and in the evaluation of cardiomyopathy [29-33]. In addition, MW can also be used to predict and evaluate therapeutic effects [34-38].

However, due to the lack of accurate reference values used for the MW indices, this method is limited to clinical research but cannot be used for routine clinical examination. Previous studies have obtained reference values of MW grouped by gender or age [7-9]. However, correlations between MW and demographic variables have been investigated, showing the absence of a strong dependence of MW indices on age or gender [7, 8]. Multivariable analysis revealed significant correlations only with SBP for MW parameters [7]. Morbach et al. observed an upward shift of GCW and GWW with advancing age [9]. This finding can be explained when considering the increase of SBP with increased age, even if the SBP remains in the normal range. The present study is the first to measure MW reference values using SBP as a grouping standard. According to our results, GWI and GCW varied greatly according to the different afterload conditions. Therefore, it is unreasonable to assess whether the MW of a given patient is in the normal range in the absence of SBP. For one thing, some patients with myocardial dysfunction will be incorrectly mistaken as normal subjects if the examination results are interpreted using the normal reference ranges previously provided by the EACVI NORRE study [7] for all the patients without taking SBP into account. For example, the normal lowest value of GWI in men was set as $1,270 \mathrm{mmHg} \%$. However, according to our results, this standard was set too low for patients with SBP higher than $100 \mathrm{mmHg}$, which can lead to misinterpretation of the examinations and result in false negative conclusions. A similar finding was noted for GCW. For another, certain studies have used PSLs to predict myocardial dysfunction and obtained the optimal cut-off values but not considered the effect of SBP on MW $[6,26]$. For example, the optimal cutoff for GWI was established as $1,810 \mathrm{mmHg} \%$ to predict significant CAD [26]. However, according to our results, it can be normal if GWI is less than $1,810 \mathrm{mmHg} \%$ as long as the SBP is low. Clearly, a low GWI due to low SBP can be mistaken for cardiac abnormality resulting in a false positive result. This reason may account for the low diagnostic specificity in that study. Therefore, the critical effect of afterload on MW cannot be ignored during clinical research or diagnosis and is required to make a reasonable judgment on the myocardial function.

Normal reference values have been reported for GWI and GCW and these indices correlated positively with SBP [7-9, 39]. However, no studies have been conducted with regard to the reference values of MW when SBP is above normal. The changes in these indices following increased afterload have not been fully investigated. It is well known that a large percentage of heart disease patients present with hypertension. Therefore, the afterload-dependent reference values are required in this subset of patients for further interpretation. According to our results (SBP of 140-189 $\mathrm{mmHg}$ ), LVEF and GLS values were preserved in hypertensive subjects without myocardial remodeling and GWI and GCW exhibited a linear association with SBP (Fig. 2). However, this finding may not apply to population with much higher blood pressure. The results demonstrated that the LV myocardium may function at higher energy levels against the increased afterload to preserve LV contractility during the compensatory phase. It is interesting to note that the results from the exercise stress echocardiography confirmed our findings. Healthy subjects demonstrated increased GWI with elevated SBP during exercise, whereas in patients with inducible ischemia, GWI did not increase and MWI was decreased in the affected segments [25, 40, 41]. True myocardial contractility was more likely to be detected under high afterload. The present study was the first to provide work reference for heart disease patients with hypertension. The potential ability to detect myocardial dysfunction under different loading conditions can be employed and assessed in future studies.

The present study demonstrated that GWE values remained constant across all SBP-groups since almost a proportional relationship was noted in both GCW and GWW. Since GWE was not affected by afterload [7, 40, 41] and had a stable reference value in all healthy subjects, it may be more suitable than other parameters as a diagnostic index of myocardial impairment. In some studies, it has been proved that GWE is the best predictor of LV myocardial contractile performance in all MW parameters $[27,28]$. However, additional work is required to assess its efficacy in other types of heart disease. 
This study has some limitations. Firstly, this was a single-center study including a limited sample size, which may not be sufficient to provide particularly accurate reference values for MW parameters. However, the present study highlighted the importance of afterload in evaluating MW by examining the association between these two parameters and emphasizing on the influence of afterload. This finding cannot be ignored in the clinical research or diagnosis and is required in order to make a reasonable judgment on the myocardial function. Secondly, the present study did not include subjects with SBP $>190 \mathrm{mmHg}$. Therefore, we could not provide the $\mathrm{MW}$ reference and establish the variation trend of MW with SBP in that population. Thirdly, it should be noted that the use of PSL did not provide a direct measure of $\mathrm{MW}$, but rather an index of this parameter due to pressure rather than wall stress being assessed in the method.

\section{Conclusions}

The amount of MW but not the work efficiency varied greatly according to the different afterload. This finding cannot be ignored during clinical research or diagnosis and is required to make a reasonable judgment on the myocardial function.

\begin{abstract}
Abbreviations
PSL: Pressure-strain loop; MW: Myocardial work; SBP: Systolic blood pressure; GWI: Global work index; GCW: Global constructive work; GWW: Global wasted work; GWE: Global work efficiency; PET: Positron emission tomography; LS: Longitudinal strain; MWI: Myocardial work index; LVP: Left ventricular pressure; LV: Left ventricular; CTA: Coronary tomography angiography; 2D: 2-Dimensional; TTE: Transthoracic echocardiography; STE: Speckle tracking echocardiography; EF: Ejection fraction; CW: Constructive work; WW: Wasted work; WE: Work efficiency; SD: Standard deviation; Cl: Confidence interval; ANOVA: Analysis of variance; CRT: Cardiac resynchronization therapy.
\end{abstract}

\section{Supplementary Information}

The online version contains supplementary material available at https://doi. org/10.1186/s12947-021-00253-2.

Additional file 1. Data.

\section{Acknowledgements}

The authors acknowledge the contribution of Songyan Liu, MS, to statistical discussion.

\section{Authors' contributions}

QL, HW contributed to literature search, study design and providing most of the patient population. LS contributed to images acquisition, data analysis and had a major contribution in writing. HF helped in the images acquisition and data analysis. TW helped in data analysis and statistical analysis. YY and DG helped in the literature search, data analysis and manuscript revise. All authors read and approved the final manuscript.

\section{Funding}

Not applicable.

\section{Availability of data and materials}

All data generated or analysed during this study are included in this published article (and its supplementary information files).

\section{Declarations}

Ethics approval and consent to participate

The study was approved by the ethics committee of China-Japan union hospital of Jilin university, and the committee's reference number was 2019022602.

\section{Consent for publication}

I have obtained consent to publish from the participant to report individual patient data.

\section{Competing interests}

The authors declare that they have no competing interests.

\section{Author details}

${ }^{1}$ Department of CT, Jilin Province FAW General Hospital, Changchun, China. ${ }^{2}$ Department of Ultrasound, China-Japan Union Hospital of Jilin University, Changchun, China. ${ }^{3}$ GE Healthcare, Clinical Education Team(CET), Pudong New Town, Shanghai, China.

Received: 24 February 2021 Accepted: 18 May 2021

Published online: 24 June 2021

\section{References}

1. Russell K, Eriksen M, Aaberge L, Wilhelmsen N, Skulstad H, Remme EW, et al. A novel clinical method for quantification of regional left ventricular pressure-strain loop area: a non-invasive index of myocardial work. Eur Heart J. 2012:33:724-33.

2. Russell K, Eriksen M, Aaberge L, Wilhelmsen N, Skulstad H, Gjesdal O, et al. Assessment of wasted myocardial work: a novel method to quantify energy loss due to uncoordinated left ventricular contractions. Am J Physiol Heart Circ Physiol. 2013;305:H996-1003.

3. Hubert A, Le Rolle V, Leclercq C, Galli E, Samset E, Casset C, et al. Estimation of myocardial work from pressure-strain loops analysis: an experimental evaluation. Eur Heart J Cardiovasc Imaging. 2018;19:1372-9.

4. Boe E, Skulstad H, Smiseth OA. Myocardial work by echocardiography: a novel method ready for clinical testing. Eur Heart J Cardiovasc Imaging. 2019;20:18-20.

5. Yingchoncharoen T, Agarwal S, Popović ZB, Marwick TH. Normal ranges of left ventricular strain: a meta-analysis. J Am Soc Echocardiogr. 2013;26:185-91.

6. Boe E, Russell K, Eek C, Eriksen M, Remme EW, Smiseth OA, et al. Noninvasive myocardial work index identifies acute coronary occlusion in patients with non-ST-segment elevation-acute coronary syndrome. Eur Heart J Cardiovasc Imaging. 2015;16:1247-55.

7. Manganaro R, Marchetta S, Dulgheru R, llardi F, Sugimoto T, Robinet $S$, et al. Echocardiographic reference ranges for normal non-invasive myocardial work indices: results from the EACVI NORRE study. Eur Heart J Cardiovasc Imaging. 2019;20:582-90.

8. Galli E, John-Matthwes B, Rousseau C, Schnell F, Leclercq C, Donal E. Echocardiographic reference ranges for myocardial work in healthy subjects: A preliminary study. Echocardiography. 2019;36:1814-24.

9. Morbach C, Sahiti F, Tiffe T, Cejka V, Eichner FA, Gelbrich G, et al. Myocardial work - correlation patterns and reference values from the population-based STAAB cohort study. PLOS ONE. 2020;15:e0239684.

10. Lancellotti P, Tribouilloy C, Hagendorff A, Popescu BA, Edvardsen T, Pierard $L A$, et al. Recommendations for the echocardiographic assessment of native valvular regurgitation: an executive summary from the European Association of Cardiovascular Imaging. Eur Heart J Cardiovasc Imaging. 2013;14:611-44.

11. Lang RM, Badano LP, Mor-Avi V, Afilalo J, Armstrong A, Ernande L, et al. Recommendations for cardiac chamber quantification by echocardiography in adults: an update from the American Society of Echocardiography 
and the European Association of Cardiovascular Imaging. Eur Heart J Cardiovasc Imaging. 2015;16:233-70.

12. Nagueh SF, Smiseth OA, Appleton CP, Byrd BF 3rd, Dokainish H, Edvardsen T, et al. Recommendations for the Evaluation of Left Ventricular Diastolic Function by Echocardiography: An Update from the American Society of Echocardiography and the European Association of Cardiovascular Imaging. Eur Heart J Cardiovasc Imaging. 2016;17:1321-60.

13. Smiseth OA, Donal E, Penicka M, Sletten OJ. How to measure left ventricular myocardial work by pressure-strain loops. Eur Heart I Cardiovasc Imaging. 2020. https://doi.org/10.1093/ehjci/jeaa301.

14. Sagawa K, Suga H, Shoukas AA, Bakalar KM. End-systolic pressure/ volume ratio: a new index of ventricular contractility. Am J Cardiol. 1977:40:748-53.

15. Suga $\mathrm{H}$. Total mechanical energy of a ventricle model and cardiac oxygen consumption. Am J Physiol. 1979;236:H498-505.

16. Hisano R, Cooper $\mathrm{G}$ 4th. Correlation of force-length area with oxygen consumption in ferret papillary muscle. Circ Res. 1987;61:318-28.

17. Forrester JS, Tyberg JV, Wyatt HL, Goldner S, Parmely WW, Swan HJ. Pressure-length loop: a new method for simultaneous measurement of segmental and total cardiac function. J Appl Physiol. 1974:37:771-5.

18. Mele D, Trevisan F, Fiorencis A, Smarrazzo V, Bertini M, Ferrari R. Current Role of Echocardiography in Cardiac Resynchronization Therapy: from Cardiac Mechanics to Flow Dynamics Analysis. Curr Heart Fail Rep. 2020:17:384-96.

19. Kostyukevich MV, van der Bijl P, Vo NM, Lustosa RP, Pio SM, Bootsma M, et al. Regional Left Ventricular Myocardial Work Indices and Response to Cardiac Resynchronization Therapy. JACC Cardiovasc Imaging. 2020;13:1852-4.

20. Prinzen FW, Lumens J. Investigating myocardial work as a CRT response predictor is not a waste of work. Eur Heart J. 2020;41:3824-6.

21. Galli E, Leclercq C, Fournet M, Hubert A, Bernard A, Smiseth OA, et al. Value of Myocardial Work Estimation in the Prediction of Response to Cardiac Resynchronization Therapy. J Am Soc Echocardiogr. 2018;31:220-30.

22. Vecera J, Penicka M, Eriksen M, Russell K, Bartunek J, Vanderheyden M, et al. Wasted septal work in left ventricular dyssynchrony: a novel principle to predict response to cardiac resynchronization therapy. Eur Heart $\rfloor$ Cardiovasc Imaging. 2016;17:624-32

23. Galli E, Leclercq C, Hubert A, Bernard A, Smiseth OA, Mabo P, et al. Role of myocardial constructive work in the identification of responders to CRT. Eur Heart J Cardiovasc Imaging. 2018;19:1010-8.

24. Tomoaia R, Beyer RS, Zdrenghea D, Dadarlat-Pop A, Cismaru G, Gusetu $G$, et al. Global work index by non-invasive pressure-strain loops: a novel parameter to assess left ventricular performance in the early stages of heart failure with preserved or mid-range ejection fraction after acute myocardial infarction. Med Ultrason. 2020. https://doi.org/10.11152/ mu-2672.

25. Borrie A, Goggin C, Ershad S, Robinson W, Sasse A. Noninvasive Myocardial Work Index: Characterizing the Normal and Ischemic Response to Exercise. J Am Soc Echocardiogr. 2020;33:1191-200.

26. Edwards NFA, Scalia GM, Shiino K, Sabapathy S, Anderson B, Chamberlain $R$, et al. Global Myocardial Work Is Superior to Global Longitudinal Strain to Predict Significant Coronary Artery Disease in Patients With Normal Left Ventricular Function and Wall Motion. J Am Soc Echocardiogr. 2019;32:947-57.

27. Qin Y, Wu X, Wang J, Li Y, Ding X, Guo D, et al. Value of territorial work efficiency estimation in non-ST-segment-elevation acute coronary syndrome: a study with non-invasive left ventricular pressure-strain loops. Int J Cardiovasc Imaging. 2020. https://doi.org/10.1007/ s10554-020-02110-1.
28. Wang RR, Tian T, Li SQ, Leng XP, Tian JW. Assessment of Left Ventricular Global Myocardial Work in Patients With Different Degrees of Coronary Artery Stenosis by Pressure-Strain Loops Analysis. Ultrasound Med Biol. 2021:47:33-42.

29. Hiemstra YL, van der Bijl P, El Mahdiui M, Bax JJ, Delgado V, Marsan NA Myocardial Work in Nonobstructive Hypertrophic Cardiomyopathy: Implications for Outcome. J Am Soc Echocardiogr. 2020;33:1201-8.

30. Galli E, Vitel E, Schnell F, Le Rolle V, Hubert A, Lederlin M, et al. Myocardial constructive work is impaired in hypertrophic cardiomyopathy and predicts left ventricular fibrosis. Echocardiography. 2019;36:74-82.

31. Roger-Rollé A, Cariou E, Rguez K, Fournier P, Lavie-Badie Y, Blanchard $V$, et al. Can myocardial work indices contribute to the exploration of patients with cardiac amyloidosis? Open Heart. 2020;7:e001346.

32. Clemmensen TS, Eiskjær H, Ladefoged B, Mikkelsen F, Sørensen J, Granstam SO, et al. Prognostic implications of left ventricular myocardial work indices in cardiac amyloidosis. Eur Heart J Cardiovasc Imaging. 2020 https://doi.org/10.1093/ehjci/jeaa097.

33. Clemmensen TS, Eiskjær H, Mikkelsen F, Granstam SO, Flachskampf FA Sørensen J, et al. Left Ventricular Pressure-Strain-Derived Myocardial Work at Rest and during Exercise in Patients with Cardiac Amyloidosis. J Am Soc Echocardiogr. 2020;33:573-82.

34. Meimoun P, Abdani S, Stracchi V, Elmkies F, Boulanger J, Botoro T, et al, Usefulness of Noninvasive Myocardial Work to Predict Left Ventricular Recovery and Acute Complications after Acute Anterior Myocardial Infarction Treated by Percutaneous Coronary Intervention. J Am Soc Echocardiogr. 2020;33:1180-90.

35. Spetsotaki K, Zayat R, Donuru S, Autschbach R, Schnoering H, Hatam N. Evaluation of Left Ventricular Myocardial Work Performance in Patients Undergoing On-Pump and Off-Pump Coronary Artery Bypass Surgery. Ann Thorac Cardiovasc Surg. 2020;26:276-85.

36. Lustosa RP, Fortuni F, van der Bijl P, Goedemans L, El Mahdiui M, MonteroCabezas JM, et al. Left ventricular myocardial work in the culprit vessel territory and impact on left ventricular remodelling in patients with ST-segment elevation myocardial infarction after primary percutaneous coronary intervention. Eur Heart J Cardiovasc Imaging. 2020. https://doi. org/10.1093/ehjci/jeaa175.

37. Bouali Y, Donal E, Gallard A, Laurin C, Hubert A, Bidaut A, et al. Prognostic Usefulness of Myocardial Work in Patients With Heart Failure and Reduced Ejection Fraction Treated by Sacubitril/Nalsartan. Am J Cardiol. 2020;125:1856-62.

38. Valentim Gonçalves A, Galrinho A, Pereira-da-Silva T, Branco L, Rio P, Timóteo AT, et al. Myocardial work improvement after sacubitril-valsartan therapy: a new echocardiographic parameter for a new treatment. J Cardiovasc Med (Hagerstown). 2020;21:223-30.

39. Manganaro R, Marchetta S, Dulgheru R, Sugimoto T, Tsugu T, llardi F, et al. Correlation between non-invasive myocardial work indices and main parameters of systolic and diastolic function: results from the EACVI NORRE study. Eur Heart J Cardiovasc Imaging. 2020;21:533-41.

40. Halabi A, Wright L, Marwick TH. Measurement of Global Myocardial Work with Exercise Testing. J Am Soc Echocardiogr. 2020;33:631-2.

41. Mansour MJ, AlJaroudi W, Mansour L, Nehme A, Hamoui O, Ayoub W, et al. Value of myocardial work for assessment of myocardial adaptation to increased afterload in patients with high blood pressure at peak exercise. Int J Cardiovasc Imaging. 2020;36:1647-56.

\section{Publisher's Note}

Springer Nature remains neutral with regard to jurisdictional claims in published maps and institutional affiliations. 